\title{
OBSERVATION OF VERY HIGH ENERGY GAMMA-RAY EMISSION FROM THE ACTIVE GALACTIC NUCLEUS 1ES 1959+650 USING THE MAGIC TELESCOPE
}

J. Albert, ${ }^{1}$ E. Aliu,${ }^{2}$ H. Anderhub,${ }^{3}$ P. Antoranz, ${ }^{4}$ A. Armada, ${ }^{2}$ M. Asensio, ${ }^{4}$ C. Baixeras,${ }^{5}$ J. A. Barrio, ${ }^{4}$ H. Bartko, ${ }^{6}$ D. Bastieri, ${ }^{7}$ W. Bednarek, ${ }^{8}$ K. Berger, ${ }^{1}$ C. Bigongiari, ${ }^{7}$ A. Biland, ${ }^{3}$ E. Bisesi, ${ }^{9}$ R. K. Bock, ${ }^{6}$ T. Bretz, ${ }^{1}$ I. Britvitch, ${ }^{3}$ M. Camara, ${ }^{4}$ A. Chilingarian, ${ }^{10}$ S. Ciprini, ${ }^{11}$ J. A. Coarasa, ${ }^{6}$ S. Commichau, ${ }^{3}$ J. L. Contreras, ${ }^{4}$ J. Cortina, ${ }^{2}$ V. Danielyan, ${ }^{10}$ F. Dazzi, ${ }^{7}$ A. De Angelis, ${ }^{9}$ R. de los Reyes, ${ }^{4}$ B. De Lotto, ${ }^{9}$ E. Domingo-Santamaría, ${ }^{2}$ D. Dorner, ${ }^{1}$ M. Doro, ${ }^{7}$ M. Errando, ${ }^{2}$ D. Ferenc, ${ }^{12}$ E. Fernández, ${ }^{2}$ R. Firpo, ${ }^{2}$ J. Flix, ${ }^{2}$ M. V. Fonseca, ${ }^{4}$ L. Font,${ }^{5}$ N. Galante ${ }^{13}$ M. Garczarczyk, ${ }^{6}$ M. Gaug, ${ }^{2}$ J. Gebauer,${ }^{6}$ R. Giannitrapani, ${ }^{9}$ M. Giller,${ }^{8}$ F. Goebel,${ }^{6}$ D. Hakobyan, ${ }^{10}$ M. Hayashida, ${ }^{6}$ T. Hengstebeck, ${ }^{14}$ D. Höhne, ${ }^{1}$ J. Hose, ${ }^{6}$ P. Jacon, ${ }^{8}$ O. Kalekin, ${ }^{14}$ D. Kranich, ${ }^{12}$ A. Laille, ${ }^{12}$ T. Lenisa, ${ }^{9}$ P. Liebing, ${ }^{6}$ E. Lindfors, ${ }^{11}$ F. Longo, ${ }^{9}$ J. López, ${ }^{2}$ M. López, ${ }^{4}$ E. Lorenz, ${ }^{6,3}$ F. Lucarelli, ${ }^{4}$ P. Majumdar, ${ }^{6}$ G. Maneva, ${ }^{15}$ K. Mannheim, ${ }^{1}$ M. Mariotti, ${ }^{7}$ M. Martínez, ${ }^{2}$ K. Mase, ${ }^{6}$ D. Mazin, ${ }^{6}$ C. Merck, ${ }^{6}$ M. Merck, ${ }^{1}$ M. Meucci, ${ }^{13}$ M. Meyer, ${ }^{1}$ J. M. Miranda, ${ }^{4}$ R. Mirzoyan, ${ }^{6}$ S. Mizobuchi, ${ }^{6}$ A. Moralejo, ${ }^{7}$ K. Nilsson, ${ }^{11}$ E. Oña-Wilhelmi, ${ }^{2}$ R. Orduña, ${ }^{5}$ N. Otte, ${ }^{6}$ I. Oya, ${ }^{4}$ D. Paneque, ${ }^{6}$ R. Paoletti, ${ }^{13}$ M. Pasanen, ${ }^{11}$ D. Pascoli, ${ }^{7}$ F. Pauss, ${ }^{3}$ N. Pavel,${ }^{14}$ R. Pegna, ${ }^{13}$ L. Peruzzo, ${ }^{7}$ A. Piccioli, ${ }^{13}$ M. Pin, ${ }^{9}$ E. Prandini, ${ }^{7}$ J. Rico, ${ }^{2}$ W. Rhode, ${ }^{16}$ B. Riegel, ${ }^{1}$ M. Rissi, ${ }^{3}$ A. Robert, ${ }^{5}$ G. Rossato, ${ }^{7}$ S. Rügamer, ${ }^{1}$ A. Saggion, ${ }^{7}$ A. Sánchez, ${ }^{7}$ P. Sartori, ${ }^{7}$ V. Scalzotto, ${ }^{5}$ R. Schmitt, ${ }^{1}$ T. Schweizer, ${ }^{14}$ M. Shayduk, ${ }^{14}$ K. Shinozaki, ${ }^{6}$ N. Sidro, ${ }^{2}$ A. Sillanpä̈̈, ${ }^{11}$ D. Sobczynska, ${ }^{8}$ A. Stamerra, ${ }^{13}$ L. Stark, ${ }^{3}$ L. Takalo, ${ }^{11}$ P. Temnikov, ${ }^{15}$ D. Tescaro, ${ }^{7}$ M. Teshima, ${ }^{6}$ N. Tonello, ${ }^{6}$ A. Torres, ${ }^{5}$ D. F. Torres, ${ }^{2}$

$$
\begin{gathered}
\text { N. Turini, }{ }^{13} \text { H. Vankov, }{ }^{15} \text { V. Vitale, }{ }^{9} \text { R. M. Wagner, }{ }^{6}{ }^{\text {T. Wibig, }}{ }^{8}{ }^{\text {W. Wittek, }}{ }^{6} \text { and J. Zapatero } \\
\text { Received } 2005 \text { August } 24 \text {; accepted } 2005 \text { November } 7
\end{gathered}
$$

\begin{abstract}
The MAGIC Cerenkov telescope has observed very high energy (VHE) $\gamma$-ray emission from the active galactic nucleus 1ES 1959+650 during $6 \mathrm{hr}$ in 2004 September and October. The observations were carried out alternating with observations of the Crab Nebula, whose data were used as a reference source for optimizing $\gamma$-ray/hadron separation and for flux comparison. The data analysis shows VHE $\gamma$-ray emission of 1ES 1959+650 with $\sim 8 \sigma$ significance, at a time of low activity in both optical and X-ray wavelengths. An integral flux above $\sim 180 \mathrm{GeV}$ of about $20 \%$ that of the Crab Nebula was obtained. The light curve, sampled over 7 days, shows no significant variations. The differential energy spectrum between $180 \mathrm{GeV}$ and $2 \mathrm{TeV}$ can be fitted with a power-law of index $-2.72 \pm 0.14$. The spectrum is consistent with the slightly steeper spectrum seen by HEGRA at higher energies, also during periods of low X-ray activity.
\end{abstract}

Subject headings: galaxies: active — galaxies: individual (1ES 1959+650) — gamma rays: observations

\section{INTRODUCTION}

\subsection{The VHE Gamma-Ray Source IES 1959+650}

The active galactic nucleus (AGN) 1ES 1959+650 is an X-ray peaking BL Lacertae object selected from the Einstein MediumSensitivity Survey (Elvis 1992). It is hosted by an elliptical galaxy at a redshift of $z=0.047$. According to the unified model of AGNs, BL Lacertae objects have relativistic jets emerging from supermassive black holes accreting at a sub-Eddington rate, viewed under a small angle of sight (Padovani \& Urry 1992). With decreasing luminosity, the peak frequency of the synchro-

\footnotetext{
1 Universität Würzburg, Am Hubland, D-97074 Würzburg, Germany.

2 Institut de Fisica d'Altes Energies, Edifici Cn., E-08193 Bellaterra, Spain.

3 Institute for Particle Physics, ETH Hönggerberg, HPK, CH-8093 Zürich, Switzerland.

${ }^{4}$ Fisica Atomica, Universidad Complutense, Avenida Complutense s/n, E-28040 Madrid, Spain.

5 Departamento de Fisica, Universitat Autonoma de Barcelona, Edifici Cc., E-08193 Bellaterra, Spain.

${ }^{6}$ Max-Planck-Institut für Physik, Föhringer Ring 6, D-80805 München, Germany.

7 Dipartimento di Fisica, Università and INFN Padova, Via Marzolo 8, I-35131 Padova, Italy.

8 Division of Experimental Physics, University of Lodz, Pomorska 149/153, PL-90236 Lodz, Poland.
}

tron emission from the relativistic jets seems to move to higher frequencies. The class of BL Lac objects in which the synchrotron peak lies in the X-ray regime are thus called HBLs (highfrequency peaked BL Lac objects). The mass of the central black hole $(\mathrm{BH})$ in $1 \mathrm{ES} 1959+650$ has been estimated to be $\sim 1.5 \times$ $10^{8} M_{\odot}$ (Falomo et al. 2002), i.e., close to the BH mass of the HBL Mrk 421, the archetype of an extragalactic very high energy (VHE) $\gamma$-ray source (Punch et al. 1992).

The first VHE $\gamma$-ray signal from 1ES 1959+650 was reported in 1998 by the Seven Telescope Array in Utah, with a $3.9 \sigma$ significance (Nishiyama et al. 2000). Observing the source in

\footnotetext{
9 Dipartimento di Fisica, Università di Udine and INFN Trieste, Via delle Scienze 208, I-33100 Udine, Italy.

10 Yerevan Physics Institute, Cosmic Ray Division, Alikhanyan Brothers Street 2, AM-375036 Yerevan, Armenia.

11 Tuorla Observatory, Väisäläntie 20, FI-21500 Piikkiö, Finland.

12 University of California, One Shields Avenue, Davis, CA 95616-8677.

13 Dipartimento di Fisica, Università di Siena and INFN Pisa, Via Roma 56, I-53100 Siena, Italy.

14 Institut für Physik, Humboldt-Universität zu Berlin, Newtonstraße 15, D-12489 Berlin, Germany.

15 Institute for Nuclear Research and Nuclear Energy, Tsarigradsko Chaussee 72, BG-1784 Sofia, Bulgaria.

${ }_{16}$ Fachbereich Physik, Universität Dortmund, Otto-Hahn-Straße 4, D-44227 Dortmund, Germany.
} 
TABLE 1

Statistics of the Raw Data Analyzed

\begin{tabular}{clcc}
\hline \hline Source & \multicolumn{1}{c}{ Date (2004) } & $\begin{array}{c}\text { Total Time } \\
(\mathrm{hr}: \text { min })\end{array}$ & $\begin{array}{c}\text { Number of Events } \\
\left(\times 10^{6}\right)\end{array}$ \\
\hline 1ES 1959+650 .................... & Sep 6-7, Oct 7, 10, 14-17 & $6: 31$ & 4.4 \\
Crab Nebula .......................... & Sep 13-16, 21-23 & $2: 17$ & 1.7 \\
Off-source............................. & Sep 8, 10-13, 17 & $2: 49$ & 2.3 \\
\hline
\end{tabular}

2000, 2001, and early 2002, the High Energy Gamma Ray Astronomy (HEGRA) collaboration reported only a marginal signal (Horns et al. 2002). In 2002 May, the X-ray flux of the source had significantly increased. Both the Whipple (Holder et al. 2003) and HEGRA (Aharonian et al. 2003) collaborations subsequently confirmed a higher VHE $\gamma$-ray flux as well. The source was also seen in 2002 by Khelifi (2002). Further high $\gamma$-ray activity periods were detected in the same year, with some flares exceeding the Crab Nebula flux by a factor of 2-3. An interesting aspect of the source activity in 2002 was the observation of a so-called orphan flare (viz., a flare of VHE $\gamma$-rays not accompanied by correlated increased activity at other wavelengths), recorded on June 4 by the Whipple collaboration (Krawczynski et al. 2004; Daniel et al. 2005). The HEGRA collaboration had observed another, less significant, orphan VHE signal during moonlight 2 days earlier (Tonello \& Kranich 2003; Tonello 2006). Both flares in VHE $\gamma$-rays, observed in the absence of high activity in X-rays, are not expected from the synchrotron self-Compton (SSC) mechanism in relativistic jets (Kellermann \& Pauliny-Toth 1969). For other HBLs, models based on the SSC mechanism (Ghisellini et al. 1998) can successfully explain most of the VHE $\gamma$-ray production. Future observations of 1ES 1959+650, therefore, are of special importance.

This paper is structured as follows: after a brief description of the MAGIC telescope, we present in $\S 2$ the data analysis using image parameters for $\gamma$-ray/hadron separation, and the reconstruction of the direction and energy of the measured photons. Results are shown in $\S 3$, comparing with data from the Crab Nebula taken around the same time and under similar zenith angles. Finally, we discuss in $\S 4$ some implications of our findings for VHE emission models and the extragalactic background light.

\subsection{The MAGIC Cerenkov Telescope}

The MAGIC telescope represents a new generation of imaging air Cerenkov telescopes (IACTs) for $\gamma$-ray astronomy. Its design has been optimized to achieve a trigger threshold lower than was possible with previous IACTs (MAGIC is eventually to reach a trigger threshold of $30 \mathrm{GeV}$ at zenith). The low threshold will make it an ideal instrument for the study of VHE $\gamma$-ray sources that have spectral cutoffs below 100-200 GeV, such as pulsars, medium-redshift AGNs, etc.

The MAGIC parameters and performance have been described elsewhere (Cortina et al. 2005; Baixeras et al. 2004). The MAGIC mirror has a diameter and focal length both of $17 \mathrm{~m}$; its camera comprises 576 hemispherical photo-multiplier tubes with diffuse lacquer coating (Paneque et al. 2004) and specially shaped light collectors, both enhancing quantum efficiency. The camera has a field of view (FOV) of $3^{\circ} .5$.

The MAGIC telescope is located on La Palma in the Canary Islands $\left(28^{\circ} .2 \mathrm{~N}, 17^{\circ} .8 \mathrm{~W}\right.$, at $2225 \mathrm{~m}$ above sea level). From this location, 1ES 1959+650 is visible from May to October under a zenith angle of $36^{\circ}$ at culmination. At a mean observation angle of $40^{\circ}$, the threshold for the physics analysis is about twice that at zenith. We present here an analysis down to $180 \mathrm{GeV}$. Past
Whipple Observatory and HEGRA observations were carried out above $700 \mathrm{GeV}$ and above $1 \mathrm{TeV}$, respectively.

\section{DATA ANALYSIS}

The analysis presented here is restricted to $\gamma$-rays with an energy above $180 \mathrm{GeV}$. At such energies, we can discriminate hadronic and electromagnetic showers using the classical techniques pioneered by the Whipple collaboration, described in Fegan (1997). The shower image in the camera is parameterized to obtain several test statistics (Hillas 1985) describing the image shape and orientation (also called image parameters or discriminant quantities). The parameters are used to reject hadronic background events by defining, in the space of these parameters, limiting values (cuts) that discriminate between $\gamma$-ray- and hadron-induced images. The parameters also permit reconstructing the arrival direction and energy of the original $\gamma$-rays.

Table 1 shows the summary of the data collected from 1ES $1959+650$, the Crab Nebula, and off-source. This period in fall 2004 corresponds to the end of the MAGIC commissioning phase. The zenith angles for these observations are all in the range $36^{\circ}-46^{\circ}$.

Generally, the Crab Nebula with its very stable flux is considered a reference source, viz., a standard candle, for VHE $\gamma$-ray astronomy. For that reason, Crab Nebula data observed with MAGIC were selected so as to match telescope operation conditions, in time and zenith angle, to those during the observation of 1ES 1959+650. So-called off-source data are collected by pointing the telescope to a sky section near the source, where no $\gamma$-ray signal is expected in the field of view. These data are used as a cross-check of the recorded cosmic-ray background.

After quality cuts (rejection of accidental triggers due to noise, etc.), and correcting for the dead time of the data acquisition system, the effective observation time for 1ES 1959+650 amounts to $\sim 6 \mathrm{hr}$. The optimal cut values of image parameters for the $\gamma$-ray/hadron separation were obtained using Monte Carlo data, ${ }^{17}$ the parameter cut values being "trained" to obtain a signal with the maximum significance from the $\sim 2 \mathrm{hr}$ of Crab Nebula data observed at the same zenith angle. These cuts were then applied to the 1ES 1959+650 data sample, without further optimization. In our analysis, we used eight image parameters; ${ }^{18}$ the optimization procedure used the "random forest" method, which optimizes the transformation of the parameter space into a single variable, called "hadronness" (Breiman 2001; Bock et al. 2004). More details on the analysis can be found in Tonello (2006).

Two of the image parameters are of particular importance: the variable SIZE, expressed as the number of photoelectrons in the camera is, for an impact parameter between $\sim 50$ and $\sim 150 \mathrm{~m}$ (equivalent to the image parameter DIST between 0.3 and $1^{\circ}$ ), to

\footnotetext{
17 The MAGIC Monte Carlo programs are based on CORSIKA 6.019; see Heck \& Knapp (2005)

${ }_{18}$ The parameters are ALPHA, SIZE, DIST, transformed WIDTH and LENGTH, two different concentration parameters, and an asymmetry parameter.
} 

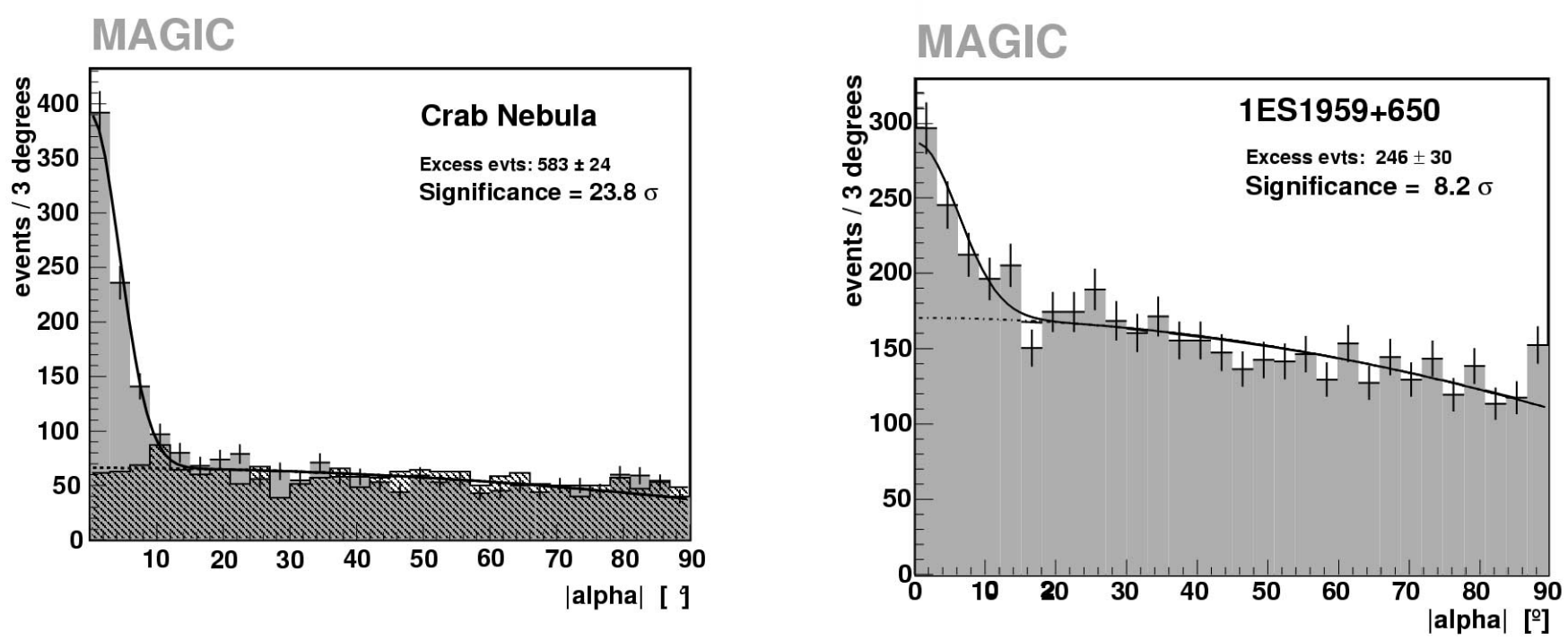

FIG. 1.-ALPHA plots of the Crab Nebula (left) and 1ES 1959+650 (right) after cuts on image parameters. Both diagrams show the second-order curve used for estimating the background at low ALPHA (up to $9^{\circ}$ ). In the left diagram (Crab Nebula), we have also added the (normalized) off-source data.

first order proportional to the energy of the incoming $\gamma$-rays; the variable ALPHA, the angle in the image between the major axis and the direction of the source, shows most clearly the existence of a signal. ALPHA is not included in the optimization process; instead, after optimizing cuts in the other parameters, we derive from the ALPHA distribution (Fig. 1) the significance of the signal (using formula [17] from Li \& Ma 1983). Finally, the data are required to satisfy low ALPHA values, thus selecting only showers that point to the source position.

\section{RESULTS}

\subsection{Alpha Plot and Comparison with Crab}

In Figure 1 (left) we show the distribution of the image parameter ALPHA for the Crab Nebula, together with the offsource data normalized to the on-source data between $20^{\circ}$ and $90^{\circ}$. Here we chose a selection of events in terms of SIZE, corresponding to a threshold $>300 \mathrm{GeV}^{19}$ In Figure 1 (right), the ALPHA distribution of the 1ES $1959+650$ data sample is shown, after applying the parameter cuts optimized using the Crab Nebula sample. The background for the Crab Nebula data un-

19 This data selection has been chosen so as to allow the application of a constant ALPHA cut of $9^{\circ}$ to the entire sample. Including lower energy events adds more background and reduces significance. The optimization of the full sample, including events of smaller SIZE, has been done separately. der the signal was estimated both from the off-source events and by extrapolating the ALPHA distribution from on-source events between $20^{\circ}$ and $90^{\circ}$, using a simple second-order formula $\left(C_{1}+C_{2} \times \mathrm{ALPHA}^{2}\right)$. Both methods give the same result; we thus used the same formula for extrapolation from the onsource events outside the excess peak for both the Crab Nebula and $1 \mathrm{ES} 1959+650$.

The significance of the 1ES $1959+650$ detection is $8.2 \sigma$, with $246 \pm 30$ excess events (after all cuts) in $\sim 6.0 \mathrm{hr}$; the signal from the Crab Nebula corresponds to $\sim 23.8 \sigma$ and $583 \pm 24$ excess events in $\sim 2.1 \mathrm{hr}$.

We obtain an integral VHE $\gamma$-ray flux from 1ES 1959+650 above $180 \mathrm{GeV}$ of $(4.7 \pm 0.5 \pm 1.6) \times 10^{-11}$ photons $\mathrm{cm}^{-2} \mathrm{~s}^{-1}$ (the errors given are statistical and an estimate for systematics, respectively). ${ }^{20}$ For the flux above $300 \mathrm{GeV}$, the result is $(1.98 \pm$ $0.21 \pm 0.38) \times 10^{-11}$ photons $\mathrm{cm}^{-2} \mathrm{~s}^{-1}$. These flux values correspond to 0.20 and 0.17 crab, respectively, when comparing to the Crab Nebula flux measured by MAGIC, and are not incompatible with the limits given by Horan et al. (2004).

We also analyzed the data set using a completely independent analysis chain, ${ }^{21}$ and obtained, within statistical limits, the same significance and flux.

\footnotetext{
${ }^{20}$ For this estimate of the flux, the small correction for the dead time of the electronics readout was not considered.

${ }^{21}$ Using dynamical supercuts as described in Kranich (1997).
}

TABLE 2

Analysis of 1ES 1959+650 Data Divided into Single Nights of Observation

\begin{tabular}{|c|c|c|c|}
\hline Date (MJD) & $\begin{array}{l}\text { Excess Events } \\
\left(\text { events minute }^{-1}\right)\end{array}$ & Significance $(\sigma)$ & $\begin{array}{c}\text { Flux }(>300 \mathrm{GeV}) \\
\left(10^{-11} \text { photoelectrons } \mathrm{cm}^{-2} \mathrm{~s}^{-1}\right)\end{array}$ \\
\hline $53254.0 \ldots \ldots \ldots \ldots \ldots \ldots \ldots \ldots \ldots \ldots \ldots \ldots \ldots \ldots$ & $0.82 \pm 0.22$ & 3.7 & $2.31 \pm 0.59$ \\
\hline 53254.9........................ & $0.54 \pm 0.24$ & 2.2 & $0.93 \pm 0.60$ \\
\hline $53285.0 \ldots \ldots \ldots \ldots \ldots \ldots \ldots \ldots \ldots \ldots \ldots \ldots \ldots \ldots \ldots$ & $0.95 \pm 0.28$ & 3.4 & $2.22 \pm 0.84$ \\
\hline $53287.9 \ldots \ldots \ldots \ldots \ldots \ldots \ldots \ldots \ldots \ldots \ldots \ldots \ldots$ & $0.95 \pm 0.30$ & 3.2 & $1.69 \pm 0.86$ \\
\hline 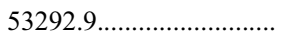 & $0.53 \pm 0.31$ & 1.7 & $1.74 \pm 0.94$ \\
\hline 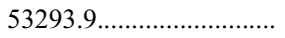 & $1.26 \pm 0.27$ & 4.7 & $3.39 \pm 0.84$ \\
\hline $53294.9 \ldots \ldots \ldots \ldots \ldots \ldots \ldots \ldots \ldots \ldots \ldots \ldots \ldots \ldots \ldots$ & $0.69 \pm 0.18$ & 3.9 & $1.55 \pm 0.50$ \\
\hline
\end{tabular}



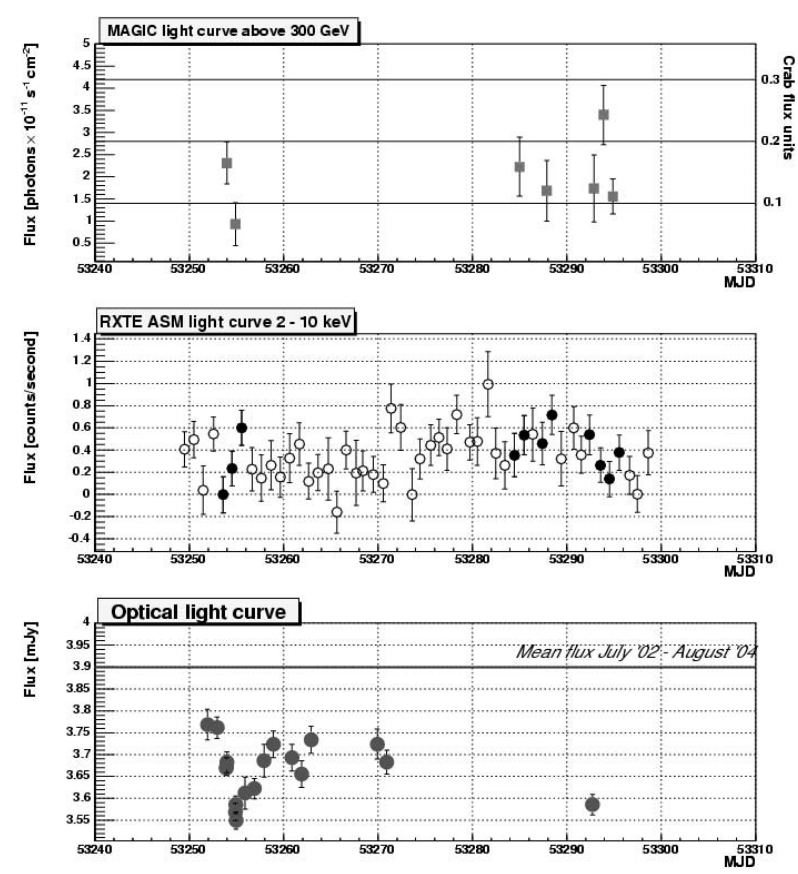

FIG. 2.-Top: Light curve as measured by MAGIC during the months of 2004 September and October. Middle: Light curve in X-rays for 1ES 1959+650 for the same period (from published RXTE ASM data). The full circles indicate those recorded during the period of $\gamma$-ray observations with MAGIC. Bottom: Optical light curve for the same period (from the Tuorla Blazar Monitoring Program). The line at $3.9 \mathrm{mJy}$ gives the average flux over nearly $2 \mathrm{yr}$ (2002 September 10 to 2004 August 25) before the MAGIC observations; during our observations, the optical activity was particularly low.

\subsection{The Light Curve, Comparing to Observations at Other Wavelengths}

Most blazars known to emit VHE $\gamma$-rays were detected at times of strong VHE $\gamma$-ray flaring, and correlated with strong $\mathrm{X}$-ray variability during the same period. For our $6 \mathrm{hr}$ observation time of 1ES 1959+650, only modest tests of the flux variation are possible. We show in Table 2 a flux analysis for each night, indicating that the source was basically in the same (low) state during the time covered by our observation; corrections for small differences in the zenith angle came out to be negligible. We show the flux again in the top diagram of Figure 2.

Strong VHE $\gamma$-ray emission from an AGN naturally raises the question if the source was also active at other wavelengths. If the $\gamma$-ray emission is due to the inverse-Compton scattering of accelerated electrons, their corresponding synchrotron emission must show up at lower energies. Most observations of other sources are indeed in line with the correlated X-ray variability expected from synchrotron self-Compton models ${ }^{22}$ (e.g., Inoue $\&$ Takahara 1996). Figures 2 and 3 show the light curves of $1 E S$ $1959+650$ in the X-ray and optical domains over MAGIC's observation period and a $3 \mathrm{yr}$ period, respectively. The X-ray data are based on published Rossi X-Ray Timing Explorer (RXTE) All-Sky Monitor (ASM) X-ray flux data;23 the optical light curve is provided by the Tuorla Observatory Blazar Monitoring Program. ${ }^{24}$ No strong activity in X-rays or the optical was observed during the period of the VHE $\gamma$-ray studies reported here. This fact and the absence of significant time variability lead to the tentative conclusion that the reported VHE $\gamma$-ray emission of

22 See the VERITAS Blazar Working Group Web site at http://jelley.wustl .edu/multiwave/spectrum/.

${ }^{23}$ Available at http:// heasarc.gsfc.nasa.gov/xteweather/.

24 Available at http://users.utu.fi/kani/1m/1ES_1959+650.html.

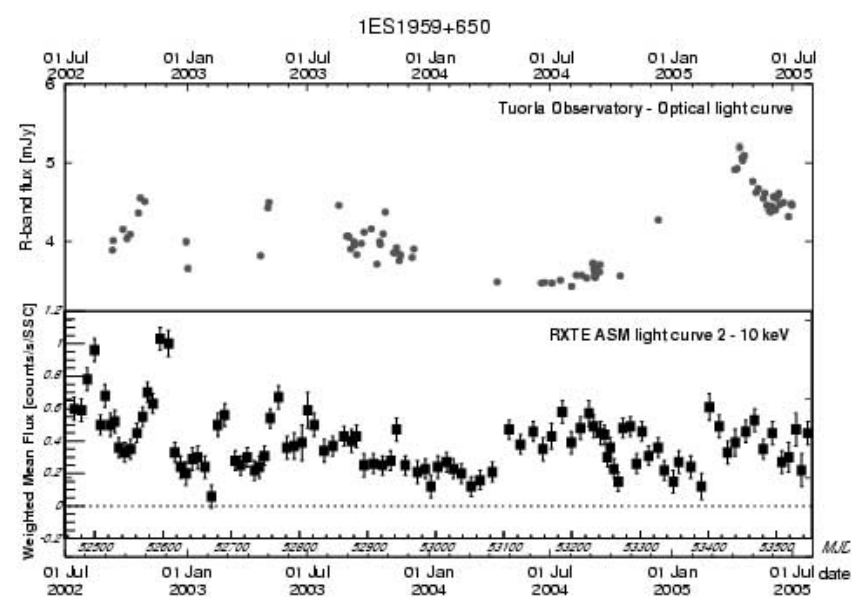

FIG. 3.-Top: Light curve in optical for 1ES 1959+650, from 2002 July to 2005 July (from the Tuorla Blazar Monitoring Program). Bottom: Light curve in the X-ray domain, over the same $3 \mathrm{yr}$ period. The points are averaged over 200 individual measurements each (from published $R X T E$ ASM data).

1ES 1959+650 does not follow the pattern observed in other AGNs during flaring periods. Future observations over longer periods will shed more light on the nature of the quiescent VHE emission of 1ES 1959+650 (see $\S 4)$.

\subsection{The VHE Gamma-Ray Spectrum and a Comparison with the Crab Nebula Spectrum}

The spectra for the Crab Nebula and 1ES 1959+650 measured in fall 2004 are shown in Figure 4. The measurements are shown, along with lines obtained by unfolding and a fit to a simple power law. Both measurements are compatible with this hypothesis, albeit with a spectral index smaller than reported at higher energies. The fits give slopes of $-2.72 \pm 0.14$ for 1 ES $1959+650$ and $-2.41 \pm 0.05$ for the Crab Nebula, respectively. There is strong evidence of the spectrum of 1ES $1959+650$ being steeper than that of the Crab Nebula over this energy range.

In Figure 5 we show a comparison with spectral data taken by HEGRA in 2002 at higher energies (Aharonian et al. 2003), with a slope of $-3.18 \pm 0.17$. The energy overlap of past and current data is small, demonstrating the progress in accessing lower

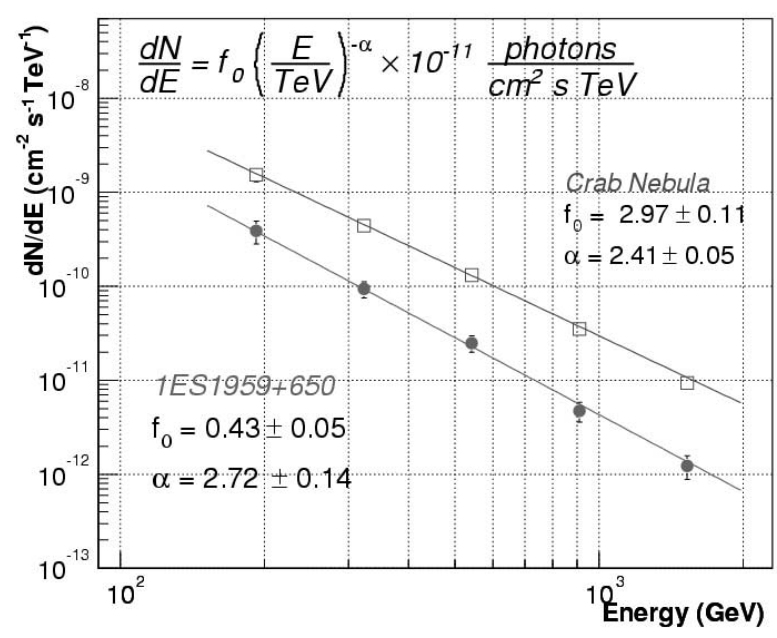

FIG. 4.-Differential spectra for the Crab Nebula and 1ES 1959+650. The energy range from $150 \mathrm{GeV}$ to $2 \mathrm{TeV}$ is divided into five bins in logarithmic scale. The point positions are the median values of the estimated energy bins, weighted with the assumed spectral slope. 


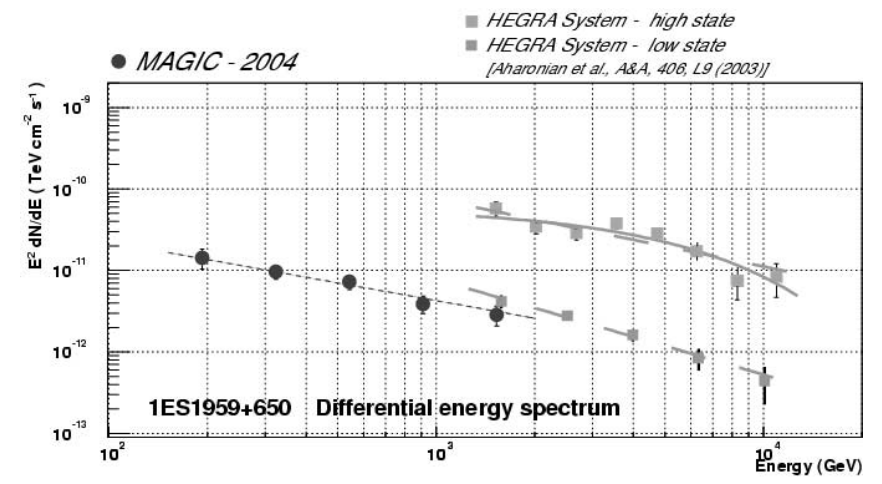

FIG. 5.-Differential spectrum for 1ES 1959+650, combined with HEGRA points from Aharonian et al. (2003). Note that the lower HEGRA points are from a comparable (low) state of activity, (although there is no unique definition of "low state"); measurements during a flaring state are also shown (larger squares).

energies with the MAGIC telescope. Past spectral descriptions required a cutoff parameter of about $3 \mathrm{TeV}$, in order to take into account possible absorption due to the cosmic infrared background. Our data are in an energy range in which the effects of such an absorption process are weak; thus, a simple power law should be sufficient to describe the data.

\section{DISCUSSION AND CONCLUSIONS}

The HBL 1ES 1959+650 has been clearly detected with the MAGIC telescope, in a few hours observation time during 2004 September and October, at a mean zenith angle of $40^{\circ}$. During that period, the source was in a quiescent state both in X-rays and at optical wavelengths. In the same period, Crab Nebula and off-source data were recorded under comparable observational conditions.

For the first time, 1ES 1959+650 has been observed down to $180 \mathrm{GeV}$, a limit much lower than that achieved in previous experiments. The energy spectrum between $180 \mathrm{GeV}$ and $2 \mathrm{TeV}$ is compatible with a power law of slope $-2.72 \pm 0.14$. A crude variability analysis over the period of observation has shown no significant variation of the $\gamma$-ray flux. The quiescent spectrum can be considered to match the spectrum measured by HEGRA at higher energy during past periods of equally low X-ray activity. We therefore tentatively conclude that a steady VHE emission component has been identified in the spectrum of 1ES 1959+650.
Explaining the observed quiescence spectrum by a one-zone SSC model is possible, but with some difficulty, since the implied relativistic electron pressure exceeds the magnetic pressure, leading to an unstable situation. SSC models, on the other hand, clearly fall short of explaining the orphan flares seen in previous observations of 1ES 1959+650 (Krawczynski et al. 2004; Daniel et al. 2005; Tonello \& Kranich 2003). The quiescence spectrum and the flares both seem to indicate the presence of an additional high-energy electron population, possibly of hadronic origin (Boettcher 2005; Mannheim 1993; Massaro et al. 2004), or proton synchrotron radiation (Aharonian 2000). Short-variability timescales might reflect dynamical effects in shock-in-jet models, or the short cooling times of protons at ultrahigh (up to $10^{19} \mathrm{eV}$ ) energies (Rachen \& Meszaros 1998).

Multiwavelength monitoring campaigns are required to further reveal the nature of the VHE emission component in 1ES $1959+650$. Such monitoring should also include future large neutrino observatories; the models based on the presence of a significant hadronic component of the 1ES $1959+650$ jet (e.g., Boettcher 2005) also predict in a natural way detectable neutrino fluxes. The AMANDA collaboration, operating a neutrino telescope in the Southern hemisphere, recently reported five recorded neutrino events from the direction of 1ES 1959+650 over a total observation period of $4 \mathrm{yr}$ (Bernardini et al. 2006). Three events coincided with 1ES 1959+650 flares; one is coincident with the orphan flare observed by the Whipple collaboration. While these observations are tantalizing, but not yet statistically compelling, they do demonstrate that neutrino astronomy has reached the stage at which fluxes at the level of the $\gamma$-ray fluxes observed with IACTs can be probed. Even for neutrino-to- $\gamma$-ray ratios smaller than unity, IceCube should soon provide the necessary experimental sensitivity (Halzen \& Hooper 2005; Ahrens et al. 2004).

We would like to thank the IAC for the excellent working conditions on the La Palma Observatory Roque de los Muchachos. We are grateful to the RXTE ASM team for their quick-look results. The support of the German BMBF and MPG, the Italian INFN, and the Spanish CICYT is gratefully acknowledged. This work was also supported by ETH research grant TH-34/04-3 and Polish grant MNiI 1P03D01028.
Aharonian, F. 2000, NewA, 5, 377

Aharonian, F., et al. 2003, A\&A, 406, L9

Ahrens, J., et al. 2004, Astropart. Phys. 20, 507

Baixeras, C., et al. 2004, Nucl. Instrum. Methods Phys. Res. A, 518, 188

Bernardini, E. 2006, in Towards a Network of Atmospheric Cherenkov Detectors VII (Palaiseau: Ecole Polytechnique), in press

Bock, R. K., et al. 2004, Nucl. Instrum. Methods Phys. Res. A, 516, 511

Boettcher, M. 2005, ApJ, 621, 176

Breiman, L. 2001, Machine Learning, 45, 5

Cortina, J., et al. 2005, in Proc. 29th International Cosmic Ray Conference (Mumbai: Tata Institute), http://icrc2005.tifr.res.in/htm/PAPERS/OG27/spacortina-J-abs1-og27-oral.pdf

Daniel, M. K., et al. 2005, ApJ, 621, 181

Elvis, M. 1992, ApJS, 80, 257

Falomo, R., et al. 2002, ApJ, 569, L35

Fegan, D. J. 1997, J. Phys. G, 23, 1013

Ghisellini, G., et al. 1998, MNRAS, 301, 451

Halzen, F., \& Hooper, D. 2005, Astropart. Phys., 23, 537

Heck, D., \& Knapp, J. 2005, Extensive Air Shower Simulation with CORSIKA: A User's Manual (version 6.204; Karlsruhe: Institut für Kernphysik), http:// www-ik.fzk.de/corsika/usersguide/usersguide.pdf

Hillas, A. 1985, in Proc. 19th International Cosmic Ray Conference, Vol. 3 (Washington: NASA), 445

\section{REFERENCES}

Holder, J., et al. 2003, ApJ, 583, L9

Horan, D., et al. 2004, ApJ, 603, 51

Horns, D., et al. 2002, in The Universe Viewed in Gamma-Rays, ed. R. Enomoto, M. Mori, \& Yanagita, S. (Tokyo: Universal Academy)

Inoue, S., \& Takahara, F. 1996, ApJ, 463, 555

Kellermann, K. I., \& Pauliny-Toth, I. I. K. 1969, ApJ, 155, L71

Khelifi, B. 2002, Ph.D. thesis, College de France, http://tel.ccsd.cnrs.fr/documents/ archives $0 / 00 / 00 / 23 / 93 /$

Kranich, D. 1997, Ph.D. thesis, Technische Univ. München

Krawczynski, H., et al. 2004, ApJ, 601, 151

Li, T., \& Ma, Y. 1983, ApJ, 272, 317

Mannheim, K. 1993, A\&A, 269, 67

Massaro, E., et al. 2004, A\&A, 422, 103

Nishiyama, T., et al. 2000, in AIP Conf. Proc. 516, 26th Int. Cosmic Ray Conf. (Salt Lake City), ed. B. Dingus, D. B. Kieda, \& M. H. Salamon (New York: AIP), 370

Padovani, P., \& Urry, M. 1992, ApJ, 387, 449

Paneque, D., et al. 2004, Nucl. Instrum. Methods Phys. Res. A, 518, 619

Punch, M., et al. 1992, Nature, 358, 477

Rachen, J. P., \& Meszaros, P. 1998, Phys. Rev. D, 58, 123005

Tonello, N. 2006, Ph.D. thesis, Technische Univ. München

Tonello, N., \& Kranich, D. 2003, in Proc. 28th Int. Cosmic Ray Conf. (Tsukuba), ed. T. Kajita et al. (Tokyo: Universal Academy), 2615 